
\title{
28 Research Square \\ Ultrasound-triggered Release of Calcein and Doxorubicin from HER2-targeted Liposomes in Breast Cancer Therapy
}

\section{Amal El Amir}

American University of Sharjah

Saniha Ajith

American University of Sharjah

Nour AlSawaftah

American University of Sharjah

Waad Abuwatfa

American University of Sharjah

Debasmita Mukhopadhyay

American University of Sharjah

Vinod Paul

American University of Sharjah

Ghaleb Husseini ( $\nabla$ ghusseini@aus.edu )

American University of Sharjah

\section{Research Article}

Keywords: Liposomes, Doxorubicin, Breast cancer, Herceptin, Ultrasound, Triggered release

Posted Date: November 20th, 2020

DOI: https://doi.org/10.21203/rs.3.rs-109604/v1

License: (c) (i) This work is licensed under a Creative Commons Attribution 4.0 International License.

Read Full License

Version of Record: A version of this preprint was published at Scientific Reports on April 6th, 2021. See the published version at https://doi.org/10.1038/s41598-021-86860-5. 


\section{Abstract}

The functionalization of liposomes with antibodies is a potential strategy to increase the specificity of liposomes and reduce side-effects of chemotherapeutic agents. The active targeting of Human Epidermal growth factor Receptor 2 (HER2) positive breast cancer cells can be achieved by coating liposomes with an anti-HER2 monoclonal antibody. In this study, we synthesized Calcein and Doxorubicin loaded immunoliposomes functionalized with the monoclonal antibody Trastuzumab. Both liposomes were characterized for size, phospholipid concentration and antibody conjugation. The effect of low-frequency ultrasound (LFUS)-induced drug release was tested using three power densities, 7.46, 9.85 and 17.31 $\mathrm{mW} / \mathrm{cm}^{2}$; and the release data were modeled using six different kinetic models. LFUS results established the sonosensitivity of both carrier types, with immunoliposomes being more acoustically sensitive than control liposomes. Results also showed an increase in the release rate as the power density increased from 7.46 to $17.31 \mathrm{~mW} / \mathrm{cm}^{2}$. Finally, the in vitro cell experiments showed enhanced uptake and cytotoxicity when breast cancer cell lines, SKBr3 and MDAMB-231, were treated with LFUS-triggered HERliposomes.

\section{Introduction}

Breast cancer is the most common type of cancer in females, constituting the second leading cause of death globally ${ }^{1,2}$. The treatment of breast cancer is complex and relies on several factors, including the type of tumor, tumor size, grade, proliferation rate and lymph node status. Existing treatment methods include surgery, chemotherapy, radiotherapy and hormonal treatments ${ }^{3}$. In recent years, the presence of breast cancer tumor markers has been investigated, and several markers were identified, including the estrogen receptor (ER), progesterone receptor (PR) and the human epidermal growth factor receptor 2 (HER2) ${ }^{3,4}$. Human epidermal growth factors belong to a family of transmembrane receptor tyrosine kinases (RTKs). HER2 is involved in important stages of growth and cell differentiation and is normally expressed at low levels in the epithelial cells of various organs such as the lungs, bladder, pancreas, breast, and prostate ${ }^{5,6}$. HER2 overexpression can lead to malignant progression, which justifies the unfavorable prognosis of breast, ovarian, gastric, and prostate cancers ${ }^{3}$. HER2 overexpression is present in approximately $25 \%$ of all breast cancers and is usually associated with more aggressive disease and endocrine therapy resistance. Studies showed that HER2 is uniformly distributed within the tumor and its expression induces significant apoptosis in breast cancer cells. These characteristics make it an appealing choice as a target in targeted therapy $3,5,6$.

One of the significant advancements in breast cancer treatment was the development of the humanized monoclonal antibody Trastuzumab, sold under the brand name Herceptin among others, which was FDA approved in 1998 for the treatment of HER2-positive breast cancers ${ }^{11-13}$. Trastuzumab can enhance the

effects of chemotherapy and reduce the risk of recurrence when used as an adjuvant therapy ${ }^{14-16}$. This immunotherapeutic is a humanized $\operatorname{lgG}(1)$ kappa monoclonal antibody $(145.5 \mathrm{kDa})$ with a high and specific affinity towards HER2 receptors ${ }^{7}$. Trastuzumab can prevent HER2 hetero-dimerization and stop 
tumor development cell signaling via several mechanisms, including reduced PI3K/Akt signaling, enhanced degradation of HER2 receptors, and antibody-dependent cellular cytotoxicity (ADCC). Hence, this drug is considered a HER2 receptor antagonist ${ }^{8-10}$.

With regard to novel cancer treatment methods, smart drug delivery systems (SDDSs) have gained prominence in the field of targeted drug delivery ${ }^{17,18}$. SDDSs are nanostructures capable of reducing drug side effects, increasing blood circulation time, and increasing drug concentrations at target sites, thus ensuring better patient compliance ${ }^{19,20}$. SDDSs release their payloads at target sites in response to internal or external triggers such as temperature, $\mathrm{pH}$, enzymes, light, mechanical waves (e.g., ultrasound) or magnetic fields ${ }^{21-25}$. Several nanostructured delivery systems have been studied for cancer treatment, such as liposomes, solid lipid nanoparticles, metal organic frameworks, micelles, dendrimers, and quantum dots ${ }^{26}$.

Liposomes are one of the most widely used nanocarriers in drug delivery ${ }^{27}$. Liposomes are nanosized to microsized concentric spheres of phospholipid bilayers separated by aqueous compartments. Liposomes assume this bilayer conformation in order to shield the hydrophobic tails of phospholipids from aqueous environments ${ }^{28,29}$. Furthermore, liposomes can be functionalized by conjugating different moieties to their surfaces ${ }^{30,31}$. For instance, the circulation-time of liposomes can be increased and their detection by the reticuloendothelial system (RES) reduced by conjugating stealth-imparting polymers to their surfaces ${ }^{32-34}$. The most widely used polymeric substance is poly-ethylene glycol (PEG). Another example of such modifications involves the conjugation of ligands to the surfaces of liposomes to enhance their selectivity and tumor targetability ${ }^{35}$. These ligands have a binding affinity toward receptors, which tend to be overexpressed on the surface of cancer cells ${ }^{5,33}$. Some commonly used targeting ligands include carbohydrates, proteins, aptamers, antibodies, and peptides ${ }^{36}$.

Liposomes that have monoclonal antibodies or antibody fragments conjugated to their surfaces are called immunoliposomes ${ }^{37,38}$. Each antibody binds to a specific antigen, making it possible to selectively target that cell, as well as reduce the undesirable interactions with healthy cells ${ }^{39}$. The presence and type of antigen vary according to the malignancy, which affects the efficacy of immunoliposomal therapy. Highly investigated targets for antibodies include the epidermal growth factor (EGFR), HER2, the vascular endothelial growth factor (VEGFR), and the prostate-specific membrane antigen (PSMA) ${ }^{40,41}$. As discussed above, once SDDSs reach their target sites, they release their contents when exposed to either an internal or external stimulus ${ }^{23}$. Several external triggers have been investigated; however, the trigger of choice in this work is ultrasound (US). US is a cyclic sinusoidal acoustic wave with frequencies higher than the human hearing range $(>20 \mathrm{kHz}){ }^{42}$. US waves have a high-pressure phase (compression) at the upper peaks and a low-pressure phase (refraction) at the lower peaks ${ }^{43,44}$. In therapeutic applications, the biological effects of US can be either thermal or mechanical ${ }^{45}$. Thermal effects result are caused by energy dissipation, while the mechanical effects occur because of the acoustic wave propagation and pressure variations ${ }^{46,47}$. The mechanical effects of US manifest as acoustic cavitation events, which is 
the formation of gas bubbles due to changes in pressure and is generally divided into stable and transient cavitation ${ }^{45}$. In stable cavitation, the bubble expands and contracts about an equilibrium radius; however, in transient cavitation, the bubble grows rapidly in size and eventually collapses violently. Cavitation depends on the intensity of the US, and occurs only when a certain threshold (determined using the mechanical index) is reached. The mechanical index (MI) is a parameter that quantifies the probability of transient cavitation happening and is calculated using the following equation $5,6,43$.

$$
M I=\frac{P_{n e g}}{\sqrt{f}}
$$

Where $P_{\text {neg }}$ is the negative pressure in $\mathrm{MPa}$, and $f$ is the frequency in $\mathrm{MHz}$.

In triggered drug delivery, the aim is to find the optimum US parameters that permit enhanced drug delivery without harming healthy cells. This could be better achieved by disrupting the nanocarriers, enhancing drug distribution in tumor tissue, or increasing the nanocarriers' cellular uptake by enhancing the cell membrane permeability ${ }^{5,48}$. On the other hand, controlled drug delivery systems are systems that release their contents at a predetermined rate, locally or systemically, for a specified period of time. In this context, mathematical modeling can provide insight into the mechanisms by which the drug is released. Common release mechanisms include: release by drug diffusion through the polymer membrane, release through the degradation of the polymer, or release through the chemical disassociation of the drug 5,49 . Release kinetics are usually influenced by the type of drug, particle size, solubility, and the amount of the drug used. In this research, six kinetic representations were used to model the low-frequency acoustic release of the model drug calcein and the chemotherapeutic drug Doxorubicin (DOX) from immunoliposomes. As will be discussed in the following sections, release was measured by monitoring the changes in fluorescence. The percent of drug released from the liposomes was represented by the Cumulative Fraction Release (CFR), as follows:

$$
C F R=\frac{I_{t}-I_{o}}{I_{\infty}-I_{o}}
$$

Where $I_{O}$ represents the baseline intensity, $I_{t}$ represents the intensity at time, $t$, and represents the highest fluorescence intensity value obtained. In this study we investigated the feasibility of enhancing DOX delivery to breast cancer cells using Herceptin and US.

\section{Materials And Methods}

\subsection{Materials}


Dipalmitoylphosphatidyl choline (DPPC) and 1,2-distearoyl-sn-glycero-3-phosphoethanolamine-N [amino (polyethylene glycol)-2000] (DSPE-PEG (2000)- $\mathrm{NH}_{2}$ ) were obtained from Avanti Polar Lipids Inc.

(Alabaster, AL, USA, supplied by Labco LLC. Dubai, UAE). Cholesterol, calcein disodium salt, L-glutamine, antibiotic solutions (penicillin and streptomycin), trypsin, fetal bovine serum (FBS), RPMI-1640 medium, Dulbecco's Phosphate Buffered Saline (DPBS) medium and the bicinchoninic acid (BCA) kit were obtained from Sigma Aldrich Chemie GmbH (Munich, Germany, supplied by Labco LLC. Dubai, UAE). Chloroform was obtained from Panreac Quimica S.A. (Spain). Doxorubicin-hydrochloride was obtained from Euroasian Transcontinental (Lower Parel, Mumbai, India). Sephadex G-100, Sephadex G-25, and Sephacryl S200 HR were obtained from Sigma-Aldrich (Munich, Germany, supplied by Labco LLC. Dubai, UAE). Trastuzumab (Herceptin ${ }^{\circledR}$ ) was obtained from a local pharmacy. 2,4,6 trichloro-1,3,5 triazine (cyanuric chloride) was obtained from Sigma-Aldrich (St. Louis, MO, US, supplied through LABCO LLC. Dubai, UAE). SKBr3 (HER2+ cells) and MDA MB-231 (HER2- cells) were purchased from American Type Culture Collection (ATCC, Manassas, VA, USA).

\subsection{Preparation of control liposomes}

Liposomes were prepared using the thin-film hydration method. Briefly, liposomes were prepared using cholesterol, DPPC, and DSPE-PEG(2000)- $\mathrm{NH}_{2}$ at molar ratios of 30:65:5, respectively. The lipids were dissolved in $4 \mathrm{ml}$ chloroform in a round-bottom flask. The chloroform was then evaporated using a rotary evaporator under vacuum at $50^{\circ} \mathrm{C}$ for 15 minutes, until a thin film was observed on the walls. Next, the lipid film was hydrated using $2 \mathrm{ml}$ of a $30-\mathrm{mM}$ calcein solution and the $\mathrm{pH}$ adjusted to 7.4. To obtain unilamellar vesicles, the solution was sonicated for 2 minutes using a $40-\mathrm{kHz}$ sonicator bath (Elma D78224, Melrose Park, IL, USA). Whereas for particle size reduction, liposomes were extruded using 200-nm polycarbonate filters (Avanti Polar Lipids, Inc., Alabaster, AL, USA). The purification of the formulation was performed using a Sephadex G-100 column (after equilibrating it with borate buffer pH 8.5). Finally, the collected fractions were stored at $4^{\circ} \mathrm{C}^{5}$.

The same protocol described above was used to prepare liposomes encapsulating ammonium sulfate instead of calcein. The ammonium sulfate method was used to load DOX into the formed liposomes. A 0.11-M solution of ammonium sulfate at a $\mathrm{pH}$ of 5.5 was prepared to hydrate the dry lipid film. Liposomes were purified with a Sephadex G-25 gel filtration column previously equilibrated with HEPES buffer (0.26 M Sucrose, $0.005 \mathrm{M}$ ascorbic acid and 0.016 M HEPES). Next, DOX was added to the liposomal solution providing a DOX to lipid ratio of 1:6 (w/w). The liposome-DOX solution was kept in a water bath at $60^{\circ} \mathrm{C}$ for 45 minutes with mild stirring. The resulting solution was then centrifuged through a Sephadex G-25 gel filtration column equilibrated with PBS $(\mathrm{pH} 7.4)^{6}$.

\subsection{Preparation of HER-liposomes}

The functionalization of the liposomes with HER was performed using a double substitution reaction. Calcein and DOX liposomes were prepared separately using the procedures detailed in the previous section. As for the functionalization procedure, cyanuric chloride was dissolved in acetone to make a 10 
$\mathrm{mg} / \mathrm{ml}$ solution, of which $9.23 \mu \mathrm{l}$ was diluted in $0.5 \mathrm{ml}$ deionized water. This solution was then added to 1 $\mathrm{ml}$ of the liposomal formulations. The reaction was conducted at a pH of 8.5 and $0{ }^{\circ} \mathrm{C}$ and was left to stir for 3 hours, allowing the nucleophilic substitution of the chloride particle on the cyanuric chloride with the proton on the $\mathrm{NH}_{2}$ group of the liposomes. The second reaction involves the linking of the $\mathrm{N}$-terminus on the amino acids of HER to cyanuric chloride. One milligram of HER was dissolved in $0.5 \mathrm{ml}$ borate buffer ( $\mathrm{pH} 8.5)$, this solution was then added to the liposomes, and the reaction was kept stirring overnight. Finally, excess HER and any free drugs were purified in a Sephacryl S-200 HR column equilibrated with PBS ( $\mathrm{pH}$ of 7.4). The liposomes were collected and stored at $4{ }^{\circ} \mathrm{C}{ }^{5,6}$.

\subsection{Particle size and polydispersity evaluation}

The particle size and polydispersity index of liposomes and immunoliposomes were measured at $25^{\circ} \mathrm{C}$. The intensity-weighted hydrodynamic radius was determined using DynaPro NanoStar (Wyatt Technology Corp., Santa Barbara, CA, USA).

\subsection{Estimation of phospholipid content}

The phospholipid content of liposomes was determined using the Stewart Assay. The mixing of a phospholipid-containing chloroform solution with ammonium ferrothiocyanate at room temperature yields a colored complex that partitions in the chloroform phase and whose maximal absorbance is 485 $\mathrm{nm}$. The liposome samples were dried under vacuum and then dissolved in chloroform. This solution was then sonicated to break the liposomes to their constituent lipids. The liposomes-chloroform solution was transferred to a centrifuge tube where $2 \mathrm{ml}$ of ammonium ferrothiocyanate was added. The centrifugation step resulted in a biphasic system; the top dark layer was removed and discarded, while the bottom clear chloroform layer was transferred to a quartz cuvette, and its optical density measured using ultraviolet-visible (UV-Vis) spectroscopy at $A_{\max }=485 \mathrm{~nm}$ against chloroform as a blank. An average of the six measurements was taken.

\subsection{Antibody conjugation efficiency in immunoliposomal formulations}

HER conjugation efficiency to liposomes was determined by the Bicinchoninic Acid Assay (BCA). The BCA reagent was prepared by mixing QuantiPro QA buffer, QuantiPro QB, and $\mathrm{CuSO}_{4}$ in a ratio of 25:25:1, respectively. One milliliter of the reagent was added to $1 \mathrm{ml}$ of PBS and $100 \mu \mathrm{l}$ of the liposomal solution, followed by incubation at $60^{\circ} \mathrm{C}$ for 1 hour. The optical density of the samples was measured using UVVis spectroscopy at $562 \mathrm{~nm}$. Moreover, the molecular weight of Trastuzumab and DPPC, in addition to the results obtained from the BCA and Stewart assay, were used to determine the number of Trastuzumab molecules per vesicle.

\subsection{Low-Frequency ultrasound release studies}

The release of calcein and DOX from liposomes was triggered using a $20-\mathrm{kHz}$ low-frequency ultrasonic probe (model VCX750, Sonics \& Materials Inc., Newtown, CT) and monitored by fluorescence changes 
using a QuantaMaster QM 30 Phosphorescence Spectrofluorometer (Photon Technology International, Edison NJ, USA). Power densities were measured using a hydrophone ((Bruel and Kjaer 8103, Decatur, $\mathrm{GA}$ ). Calcein is a fluorescent molecule with excitation and emission wavelengths of 495 and $515 \mathrm{~nm}$, respectively. As for DOX the emission wavelength is $595 \mathrm{~nm}$, while its excitation wavelength is around $485 \mathrm{~nm}$. The sample to be tested was prepared by diluting $75 \mu \mathrm{L}$ of liposomes in $3 \mathrm{~mL}$ of PBS in a fluorescence cuvette. The initial fluorescence intensity $I_{O}$ was measured for 60 seconds before sonication. Then, US was applied in a pulsed mode, with 20 seconds on and 10 seconds off for calcein liposomes and 20 seconds on, 20 seconds off for DOX liposomes to account for the thermal effects of US. The release was performed using three different power densities, $7.46,9.85$, and $17.31 \mathrm{~mW} / \mathrm{cm}^{2}$. The pulsed US cycles mentioned earlier were continued until a fluorescence plateau was reached, at which point 50 $\mu \mathrm{L}$ of Triton X-100 (Tx100) were added to the sample to lyse liposomes and release all the encapsulated contents, simulating $100 \%$ drug release.

\subsection{MTT assay}

SKBr3 (HER2+ cells) and MDA MB-231 (HER2- cells) were cultured in DMEM and RPMI media, respectively, supplemented with $10 \%$ Fetal Bovine Serum and $1 \%$ penicillin/streptomycin. Cells were grown at $37^{\circ} \mathrm{C}$ under $5 \% \mathrm{CO}_{2}$. Aliquots containing $1 \times 10^{4}$ cells per well were seeded into 96 -well plates and incubated for 24 hours prior to treatment to ensure proper cellular confluency. After 24 hours, different treatments were added in triplicates to the cells with a concentration of $8 \mu \mathrm{M}$ per well. The cells were treated with free DOX, DOX-loaded liposomes and DOX-HER liposomes, then incubated for 5 hours. Following incubation, the plates were subjected to continuous LFUS for 20 seconds in a $35-\mathrm{kHz}$ bath. One plate of each cell line was not subjected to US to serve as a control. Next, the plates were incubated for 48 hours, after which the culture medium was replaced with the MTT medium, containing $20 \mu \mathrm{l}$ of the sterile MTT dye $(5 \mathrm{mg} / \mathrm{ml})$, and further incubated at $37^{\circ} \mathrm{C}$ for $4 \mathrm{~h}$. After the incubation, $100 \mu \mathrm{l}$ of DMSO (Sigma, USA) was mixed with the medium and incubated for $15 \mathrm{~min}$. Finally, absorbance values were read using a microplate reader (AccuReader, Nangang, Taipei, Metertech, Taiwan) at $570 \mathrm{~nm}$. For each group, three replicates were analyzed, and a mean value was estimated. The cell viability was calculated by dividing the optical density (OD) value of the experimental group by the mean OD of the control group and multiplying it by $100 \%$.

\subsection{Flow cytometry analysis}

For the flow cytometry assay, both SKBR-3 and MDA-MB-231 cells $\left(2 \times 10^{5}\right.$ cells $\left./ \mathrm{ml}\right)$ were seeded in 6-well plates for $24 \mathrm{~h}$. On the following day, the cells were treated with the control and HER-DOX liposomes and further incubated for 3.5 hours, after which the cells were sonicated with a LFUS bath at a frequency of $35 \mathrm{kHz}$ for $45 \mathrm{sec}$ and a power density of $20 \mathrm{~mW} / \mathrm{cm}^{2}$. One plate from each cell line was treated with both types of liposomes but was not exposed to US to serve as a reference. Following sonication, the cells were again incubated for $1 \mathrm{~h}$. Trypsin was then added to the plates to detach the cells, which were resuspended in PBS to prepare them for the flow cytometer analysis. 


\subsection{Statistical Analysis}

Results were reported as average \pm standard deviation (SD). One-way ANOVA tests were used to compare the sizes of the control and immunoliposomes; while two-factor ANOVA tests were employed to analyze LFUS release results and kinetic modeling findings. Both types of ANOVA tests assumed that both populations have similar variances, and two values are considered statistically different if $p<0.05$ and if $\mathrm{F}<\mathrm{F}_{\text {critical }}$ (unless otherwise stated).

\section{Results And Discussion}

\subsection{Liposomes characterization}

Dynamic light scattering (DLS) measurements were performed to ensure the formation of liposomes and to measure the size of both types of synthesized liposomes. A polydispersity $(\mathrm{Pd})$ index upper limit of $20 \%$ is generally acceptable for DLS measurements. Table 1 summarizes the average sizes of the three batches of liposomes with their respective standard deviations. Based on these findings, both types of liposomes (both calcein and DOX-loaded) are well within the size range for the EPR effects to take place.

Table 1: Size and polydispersity of Calcein and DOX-loaded control and immunoliposomes.

\begin{tabular}{|c|c|c|c|c|}
\hline \multirow{2}{*}{ Liposomes } & \multicolumn{2}{|c|}{ Radius (nm) } & \multicolumn{2}{c|}{ pd\% } \\
\cline { 2 - 5 } & Calcein & DOX & Calcein & DOX \\
\hline $\mathrm{NH}_{2}$ liposomes & $89.54 \pm 0.50$ & $91.2 \pm 1.47$ & $11.28 \pm 1.11$ & $12.3 \pm 3.01$ \\
\hline HER liposomes & $101.10 \pm 1.13$ & $94.9 \pm 1.29$ & $17.22 \pm 2.34$ & $11.2 \pm 0.55$ \\
\hline
\end{tabular}

A one-way ANOVA analysis was conducted to compare the radii of the $\mathrm{NH}_{2}$ liposomes and the immunoliposomes. The results showed a higher $F$-value compared to $F_{\text {critical, }}$ as well as a $p$-value lower than the standard alpha value of 0.05 , which indicated that the two types of nanoparticles had statistically different radii.

To confirm the conjugation of Trastuzumab to liposomes, the results from the BCA and Stewart assays were used. As mentioned above, the BCA assay was used to determine the protein concentration in the samples in $\mu \mathrm{g} / \mathrm{ml}$, while the Stewart assay was used to determine the DPPC concentration in $\mathrm{mg} / \mathrm{l}$. According to the BCA results, HER-liposomes exhibited a 1.5-fold increase in protein concentration compared to control liposomes (refer Figure 1). Combining the findings of both assays yielded a weightto-weight $(\mathrm{w} / \mathrm{w})$ ratio of proteins to lipids in $\mu \mathrm{g} / \mathrm{mg}$. The results of three batches were averaged to confirm conjugation consistency and calculate the standard deviation. Therefore the average number of lipid molecules constructing a single liposome vesicle would be $80,000^{50}$. Knowing the concentration of Trastuzumab and the lipids making up the liposomes, as well as their respective molecular weights, it was found that almost 9 Trastuzumab molecules were conjugated per liposome, which is considered well within the optimal range necessary to induce sufficient cells cytotoxicity ${ }^{51}$.

\subsection{Low-frequency ultrasound (LFUS) release studies}


LFUS release was performed on three batches of control liposomes and immunoliposomes (with three replicate measurements for each batch). As mentioned above, US was applied in a pulsed mode, with 20 seconds on and 10 seconds off for calcein liposomes and 20 seconds on, 20 seconds off for DOX liposomes. The plateau after every pulse signifies no release when ultrasound is "off". The release trends for calcein control liposomes are depicted in Figure 2 and Table 2. Figure 2 shows that as the power density increases, the release rate becomes steeper, which is expected due to an increase in cavitation events with increased power density. It can also be seen that the control liposomes released most of their contents ( $85 \%)$ within 3 minutes, indicating high sonosensitivity. Additionally, the release trend at 17.31 $\mathrm{mW} / \mathrm{cm}^{2}$ can be seen to decrease slightly after the plateau. This behavior may be attributed to the thermal effects experienced by liposomes.

Table 2. Summary of release results for calcein liposomes.

\begin{tabular}{|c|c|c|}
\hline Liposome type & Power Density $\left(\mathrm{mW} / \mathrm{cm}^{2}\right)$ & CFR at plateau \\
\hline Control liposomes & 7.46 & 0.8530 \\
\cline { 2 - 3 } & 9.85 & 0.8820 \\
\cline { 2 - 3 } & 17.31 & 0.8554 \\
\hline HER liposomes & 7.46 & 0.9109 \\
\cline { 2 - 3 } & 9.85 & 0.9257 \\
\cline { 2 - 3 } & 17.31 & 0.9204 \\
\hline
\end{tabular}

Similar results were obtained for the LFUS release from immunoliposomes. As observed with control liposomes, the release rate of immunoliposomes also increased with increasing power density. Figure 2 and Table 2 illustrate the normalized online release rate of calcein from immunoliposomes, at the three investigated power densities, for three batches of liposomes. The number of pulses needed to release the Trastuzumab liposomal contents decreased with increasing power density, which can be explained by the increase in cavitation events as the power density increased. In addition, as with control liposomes, the release profile can be seen to decrease slightly after the plateau at the power density of $17.31 \mathrm{~mW} / \mathrm{cm}^{2}$, which again can be attributed to the thermal effects.

When comparing the LFUS release behavior of the control liposomes and immunoliposomes, it can be noted that both types of liposomes demonstrated what could be considered "ideal" release profiles for controlled drug release systems. However, immunoliposomes were more acoustically sensitive, releasing around $92 \%$ of the model drug, compared to approximately $86 \%$ released from control liposomes. We attribute this behavior to the presence of the antibody, which appears to slightly destabilize the 
membrane making the liposomes more susceptible to acoustic mechanical waves. This enhanced sensitivity of immunoliposomes to US waves is advantageous because therapeutic drug levels could be reached in shorter exposure times.

Similar results were obtained with the release from DOX-loaded liposomes. The release increased with increasing power density for both types of liposomes, and HER-DOX liposomes showed higher sonosensitivity. Figure 3 and Table 3 summarize the findings for both control and HER-DOX liposomes.

Table 3. Summary of release results for DOX-liposomes.

\begin{tabular}{|c|c|c|}
\hline Liposome type & Power Density $\left(\mathbf{m W} / \mathrm{cm}^{2}\right)$ & CFR at plateau \\
\hline Control liposomes & 7.46 & 0.6810 \\
\cline { 2 - 3 } & 9.85 & 0.7411 \\
\cline { 2 - 3 } & 17.31 & 0.8097 \\
\hline HER liposomes & 7.46 & 0.9041 \\
\cline { 2 - 3 } & 9.85 & 0.9326 \\
\cline { 2 - 3 } & 17.31 & 0.9312 \\
\hline
\end{tabular}

Finally, the mechanical index (MI), is a parameter used to indicate the possibility of the occurrence of cavitation. The negative pressure (expressed in units of $\mathrm{MPa}$ ) in the above equation is dependent upon the acoustic impedance of water, $Z$, and the intensity of the LFUS, $I$, (expressed in W/ $\mathrm{cm}^{2}$ ). Negative pressure is denoted by equation (3).

$$
P_{n e g}=\sqrt{2 I Z}
$$

Since the acoustic impedance of soft body tissues is comparable to that of water; hence, when determining $P_{\text {neg, }}$ the acoustic impedance of water, i.e., $1.48 \mathrm{MPa} \mathrm{sec} / \mathrm{m}$ was used ${ }^{52,53}$. The LFUS power densities used in this research were $7.46,9.85$, and $17.31 \mathrm{~mW} / \mathrm{cm}^{2}$, corresponding to MI values of 0.11 , 0.12 , and 0.16 , respectively. The threshold of collapse cavitation is expected to occur at around $\mathrm{Ml}=0.3$, biological effects are observed at $\mathrm{Ml}>0.7$, and tissue damage is expected to occur at $\mathrm{Ml}>1^{54-57}$. Therefore, the obtained values are well below the collapse cavitation threshold of 0.3 , indicating the occurrence of stable cavitation.

\subsection{In-vitro cytotoxicity analysis}


To assess the therapeutic efficiency of DOX encapsulated HER-liposomes, the MTT assay was performed using both HER-positive (HER+) and HER-negative (HER-) breast cancer cells. SKBr3 (HER2+ cells) and MDA MB-231 (HER2- cells) cells were treated with free DOX, DOX-loaded control liposomes and DOXloaded HER-liposomes at a final DOX concentration of $8 \mu \mathrm{M}$ for $5 \mathrm{~h}$, followed by continuous LFUS in a 35$\mathrm{kHz}$ sonicating bath. The MTT results revealed the enhanced efficacy of the liposomal formulations upon cell exposure to US. Free DOX at a cytotoxic concentration $(8 \mu \mathrm{M})$ was used as a positive control. The HER2+ cells viability \% treated with HER-DOX liposomes was found to be $39 \pm 0.86 \%$, and further dropped to $27 \pm 0.26 \%$ after US treatment (refer Figure 4A). In the HER2- cell line, the performance of targeted liposomes was similar to the control ones; however, more cell death was induced in the cells exposed to US (refer Figure 4B).

\subsection{Cellular uptake study by flow cytometry analysis}

Flow cytometry analysis was performed to compare the DOX uptake by two breast cancer cells SKBr3 (HER2+) and MDA-MB-231 (HER2-). Since HER2 receptors are overexpressed on the surface of SKBr3 cell lines, the intake of DOX when treated with DOX-loaded HER- liposomes was $600 \%$ higher compared to control liposomes. This was further improved by the application of US, as a $1000 \%$ improvement was observed compared to the control liposomes, refer Figure 5.

\section{Conclusion}

Smart drug delivery systems (SDDS), particularly liposomes, are an effective strategy in cancer treatment; by enhancing the pharmacokinetics and bio-distribution of chemotherapeutic agents, these nanocarriers allow the drug to interact with cancer cells, thus reducing unwanted side effects. The selectivity of liposomes can be further enhanced by conjugating targeting moieties to their surface. Once at the tumor site, a stimulus, such as ultrasound, can be used to trigger the release of the drug. This research reports on the synthesis of immunoliposomes, encapsulating the model drug/dye calcein and chemotherapeutic drug doxorubicin to target a HER-positive breast cancer cell line and studies the effects of low-frequency ultrasound, applied at different power densities, on payload release. The monoclonal antibody, Trastuzumab, was selected as a targeting moiety due to its high affinity toward HER2 breast cancer. Control liposomes and immunoliposomes (both Calcein and DOX-loaded) were successfully synthesized using the thin-film hydration method. The phospholipid content and moiety attachment were confirmed using the Stewart and BCA assays, respectively. Both types of liposomes were tested for their release under LFUS at $20 \mathrm{kHz}$. The liposomal formulations were nano in size and were small enough to extravasate at the tumor site via the EPR effect.

The quantification of phospholipids and antibodies yielded a count of nine Trastuzumab molecules per liposome, which was considered enough to induce the affinity effect needed to target the HER2 overexpressed on breast cancer cells. Moreover, short exposure to LFUS effectively released the model drug calcein and the chemotherapeutic agent DOX from both types of liposomes, thereby improving the therapeutic efficacy significantly. Immunoliposomes were found to be more acoustically sensitive than 
control liposomes. Additionally, in vitro cytotoxicity of DOX-loaded HER-liposomes against HER positive and negative breast cancer cells showed excellent cytotoxic efficacy.

In summary, this research reports important findings of utilizing ultrasound and immunoliposomes in the treatment of breast cancer. Testing the release at higher frequencies and intensities can provide additional insights into the best way to implement drug delivery in-vivo and in clinical settings.

\section{Declarations}

\section{Acknowledgments}

This work was financially supported by the American University of Sharjah Faculty Research Grants, AlJalila Foundation (AJF 2015555), Al Qasimi Foundation, Patient's Friends Committee-Sharjah, Takamul, the Technology Innovation Pioneer (TIP) Healthcare awards, and Dana Gas Endowed Chair for Chemical Engineering.

\section{Author Contribution}

A. E., S. A., D. M. and W. A. performed the experiments, and A. E., S. A., D. M., W. A., N. A., and G. H. performed the analyses. All authors discussed the results and contributed to the writing of the final version of the manuscript.

\section{Additional information}

No potential conflict of interest was reported by the authors.

\section{References}

1. Torre, L. A., Islami, F., Siegel, R. L., Ward, E. M. \& Jemal, A. Global cancer in women: Burden and trends. Cancer Epidemiology Biomarkers and Prevention vol. 26 444-457 (2017).

2. Azamjah, N., Soltan-Zadeh, Y. \& Zayeri, F. Global trend of breast cancer mortality rate: A 25-year study. Asian Pacific J. Cancer Prev. 20, 2015-2020 (2019).

3. Eloy, J. O., Petrilli, R., Brueggemeier, R. W., Marchetti, J. M. \& Lee, R. J. Rapamycin-loaded Immunoliposomes Functionalized with Trastuzumab: A Strategy to Enhance Cytotoxicity to HER2positive Breast Cancer Cells. Anticancer. Agents Med. Chem. 17, 48-56 (2017).

4. Eloy, J. O. et al. Anti-HER2 immunoliposomes for co-delivery of paclitaxel and rapamycin for breast cancer therapy. Eur. J. Pharm. Biopharm. 115, 159-167 (2017).

5. Ahmed, A. E. E. Ultrasoud Triggered Release of Trastuzumab-conjugated Immunoliposomes Targeting Breast Cancer. (American University of Sharjah, 2018).

6. Ajith, S. A Novel Cancer Treatment Platform Utilizing HER2-Immunoliposomes and Ultrasound. (American University of Sharjah, 2020). 
7. Iqbal, N. \& Iqbal, N. Human Epidermal Growth Factor Receptor 2 (HER2) in Cancers: Overexpression and Therapeutic Implications. Mol. Biol. Int. 2014, 1-9 (2014).

8. Tai, W., Mahato, R. \& Cheng, K. The role of HER2 in cancer therapy and targeted drug delivery. Journal of Controlled Release vol. 146 264-275 (2010).

9. Maadi, H., Nami, B., Tong, J., Li, G. \& Wang, Z. The effects of trastuzumab on HER2-mediated cell signaling in $\mathrm{CHO}$ cells expressing human HER2. BMC Cancer 18, (2018).

10. Nahta, R. Molecular Mechanisms of Trastuzumab-Based Treatment in HER2-Overexpressing Breast Cancer. ISRN Oncol. 2012, 1-16 (2012).

11. FDA approves new treatment option for patients with HER2-positive breast cancer who have progressed on available therapies | FDA.

12. Wong, D. J. L. \& Hurvitz, S. A. Recent advances in the development of anti-HER2 antibodies and antibody-drug conjugates. Annals of Translational Medicine vol. 2122 (2014).

13. Pernas, S. \& Tolaney, S. M. HER2-positive breast cancer: new therapeutic frontiers and overcoming resistance. Therapeutic Advances in Medical Oncology vol. 11 (2019).

14. Kennedy, S. P. et al. Targeting promiscuous heterodimerization overcomes innate resistance to ERBB2 dimerization inhibitors in breast cancer. Breast Cancer Res. 21, 43 (2019).

15. Wang, J. \& Xu, B. Targeted therapeutic options and future perspectives for her2-positive breast cancer. Signal Transduction and Targeted Therapy vol. 4 (2019).

16. Cameron, D. et al. 11 years' follow-up of trastuzumab after adjuvant chemotherapy in HER2-positive early breast cancer: final analysis of the HERceptin Adjuvant (HERA) trial. Lancet 389, 1195-1205 (2017).

17. Mitra, A. K. et al. Novel delivery approaches for cancer therapeutics. J. Control. Release 219, 248268 (2015).

18. Kalaydina, R. V., Bajwa, K., Qorri, B., Decarlo, A. \& Szewczuk, M. R. Recent advances in "smart" delivery systems for extended drug release in cancer therapy. Int. J. Nanomedicine 13, 4727-4745 (2018).

19. Phillips, M. A., Gran, M. L. \& Peppas, N. A. Targeted nanodelivery of drugs and diagnostics. Nano Today vol. 5 143-159 (2010).

20. Oliveira, M. F., Guimarães, P. P. G., Gomes, A. D. M., Suárez, D. \& Sinisterra, R. D. Strategies to target tumors using nanodelivery systems based on biodegradable polymers, aspects of intellectual property, and market. Journal of Chemical Biology vol. 6 7-23 (2013).

21. Gong, X., Wang, H. \& Yuan, Y. Analysis of the first therapeutic-target-achieving time of warfarin therapy and associated factors in patients with pulmonary embolism. Exp. Ther. Med. 12, 22652274 (2016).

22. Pharmacokinetics: The Dynamics of Drug Absorption, Distribution, Metabolism, and Elimination I Goodman \& Gilman's: The Pharmacological Basis of Therapeutics, $13 \mathrm{e} \mid$ AccessHemOnc | McGrawHill Medical. 
23. Karimi, M. et al. Smart micro/nanoparticles in stimulus-responsive drug/gene delivery systems. Chemical Society Reviews vol. 45 1457-1501 (2016).

24. Hossen, S. et al. Smart nanocarrier-based drug delivery systems for cancer therapy and toxicity studies: A review. Journal of Advanced Research vol. 15 1-18 (2019).

25. Wang, Y. \& Kohane, D. S. External triggering and triggered targeting strategies for drug delivery. Nature Reviews Materials vol. 2 1-14 (2017).

26. Patra, J. K. et al. Nano based drug delivery systems: Recent developments and future prospects 10 Technology 1007 Nanotechnology 03 Chemical Sciences 0306 Physical Chemistry (incl. Structural) 03 Chemical Sciences 0303 Macromolecular and Materials Chemistry 11 Medical and He. Journal of Nanobiotechnology vol. 1671 (2018).

27. Gao, W., Hu, C. M. J., Fang, R. H. \& Zhang, L. Liposome-like nanostructures for drug delivery. J. Mater. Chem. B 1, 6569-6585 (2013).

28. Pandey, H., Rani, R. \& Agarwal, V. Liposome and their applications in cancer therapy. Brazilian Arch. Biol. Technol. 59, 16150477 (2016).

29. Akbarzadeh, A. et al. Liposome: Classification, preparation, and applications. Nanoscale Res. Lett. 8 , 102 (2013).

30. Riaz, M. K. et al. Surface functionalization and targeting strategies of liposomes in solid tumor therapy: A review. Int. J. Mol. Sci. 19, (2018).

31. Eroğlu, İ. \& ibrahim, M. Liposome-ligand conjugates: a review on the current state of art. Journal of Drug Targeting vol. 28 225-244 (2020).

32. Li, S.-D. \& Huang, L. Nanoparticles evading the reticuloendothelial system: Role of the supported bilayer. Biochim. Biophys. Acta - Biomembr. 1788, 2259-2266 (2009).

33. Nag, O. K. \& Awasthi, V. Surface engineering of liposomes for stealth behavior. Pharmaceutics $\mathbf{5}$, 542-569 (2013).

34. Allen, T. M. The use of glycolipids and hydrophilic polymers in avoiding rapid uptake of liposomes by the mononuclear phagocyte system. Advanced Drug Delivery Reviews vol. 13 285-309 (1994).

35. Suk, J. S., Xu, Q., Kim, N., Hanes, J. \& Ensign, L. M. PEGylation as a strategy for improving nanoparticle-based drug and gene delivery. Advanced Drug Delivery Reviews vol. 99 28-51 (2016).

36. Deshpande, P. P., Biswas, S. \& Torchilin, V. P. Current trends in the use of liposomes for tumor targeting. Nanomedicine vol. 8 1509-1528 (2013).

37. Messerschmidt, S. K. E., Beuttler, J. \& Rothdiener, M. Immunoliposomes. in Antibody Engineering 401-416 (Springer Berlin Heidelberg, 2010). doi:10.1007/978-3-642-01147-4_31.

38. Park, J. W., Benz, C. C. \& Martin, F. J. Future directions of liposome- and immunoliposome-based cancer therapeutics. Semin. Oncol. 31, 196-205 (2004).

39. Chaplin, D. D. Overview of the immune response. J. Allergy Clin. Immunol. 125, S3 (2010).

40. Tsui, P., Rubenstein, M. \& Guinan, P. Correlation between PSMA and VEGF expression as markers for LNCaP tumor angiogenesis. J. Biomed. Biotechnol. 2005, 287-290 (2005). 
41. Kluetz, P. G., Figg, W. D. \& Dahut, W. L. Angiogenesis inhibitors in the treatment of prostate cancer. Expert Opinion on Pharmacotherapy vol. 11 233-247 (2010).

42. Miller, D. L. et al. Overview of therapeutic ultrasound applications and safety considerations. Journal of Ultrasound in Medicine vol. 31 623-634 (2012).

43. Schroeder, A., Kost, J. \& Barenholz, Y. Ultrasound, liposomes, and drug delivery: principles for using ultrasound to control the release of drugs from liposomes. Chem. Phys. Lipids 162, 1-16 (2009).

44. Frenkel, V. Ultrasound mediated delivery of drugs and genes to solid tumors. Adv. Drug Deliv. Rev. 60, 1193-1208 (2008).

45. Izadifar, Z., Babyn, P. \& Chapman, D. MECHANICAL AND BIOLOGICAL EFFECTS OF ULTRASOUND: A REVIEW OF PRESENT KNOWLEDGE. (2017) doi:10.1016/j.ultrasmedbio.2017.01.023.

46. Thermal Dissipation - an overview / ScienceDirect Topics.

47. O'Brien, W. D. Ultrasound-biophysics mechanisms. Progress in Biophysics and Molecular Biology vol. 93 212-255 (2007).

48. Elkhodiry, M. A. et al. Synergistic Nanomedicine: Passive, Active, and Ultrasound-Triggered Drug Delivery in Cancer Treatment. J. Nanosci. Nanotechnol. 16, 1-18 (2016).

49. Dash, S., Murthy, P. N., Nath, L. \& Chowdhury, P. Kinetic modeling on drug release from controlled drug delivery systems. Acta Pol. Pharm. 67, 217-23.

50. Kirpotin, D. et al. Sterically Stabilized Anti-HER2 Immunoliposomes: Design and Targeting to Human Breast Cancer Cells in vitro. Biochemistry 36, 66-75 (1997).

51. Shmeeda, H., Tzemach, D., Mak, L. \& Gabizon, A. Her2-targeted pegylated liposomal doxorubicin: Retention of target-specific binding and cytotoxicity after in vivo passage. J. Control. Release 136, 155-160 (2009).

52. University of New South Wales. Acoustic impedance, intensity and power. http://www.animations.physics.unsw.edu.au/jw/sound-impedance-intensity.htm.

53. Husseini, G. A., Diaz De La Rosa, M. A., Richardson, E. S., Christensen, D. A. \& Pitt, W. G. The role of cavitation in acoustically activated drug delivery. J. Control. Release 107, 253-261 (2005).

54. Stringham, S. B. et al. Over-pressure suppresses ultrasonic-induced drug uptake. Ultrasound Med. Biol. 35, 409-15 (2009).

55. Staples, B. J. et al. Role of frequency and mechanical index in ultrasonic-enhanced chemotherapy in rats. Cancer Chemother. Pharmacol. 64, 593-600 (2009).

56. Azhari, H. Basics of biomedical ultrasound for engineers. (Wiley, 2010).

57. Apfel, R. E. \& Holland, C. K. Gauging the likelihood of cavitation from short-pulse, low-duty cycle diagnostic ultrasound. Ultrasound Med. Biol. 17, 179-185 (1991).

58. Costa, P. \& Sousa Lobo, J. M. Modeling and comparison of dissolution profiles. Eur. J. Pharm. Sci. 13, 123-133 (2001).

59. Paarakh, M. P., Jose, P. A. N. I., Setty, C. M. \& Peter, G. V. Release Kinetics - Concepts and Applications. Int. J. Pharm. Res. Technol. 10, 1-9 (2018). 
60. Siepmann, J. \& Siepmann, F. Mathematical modeling of drug delivery. Int. J. Pharm. 364, 328-343 (2008).

Figures

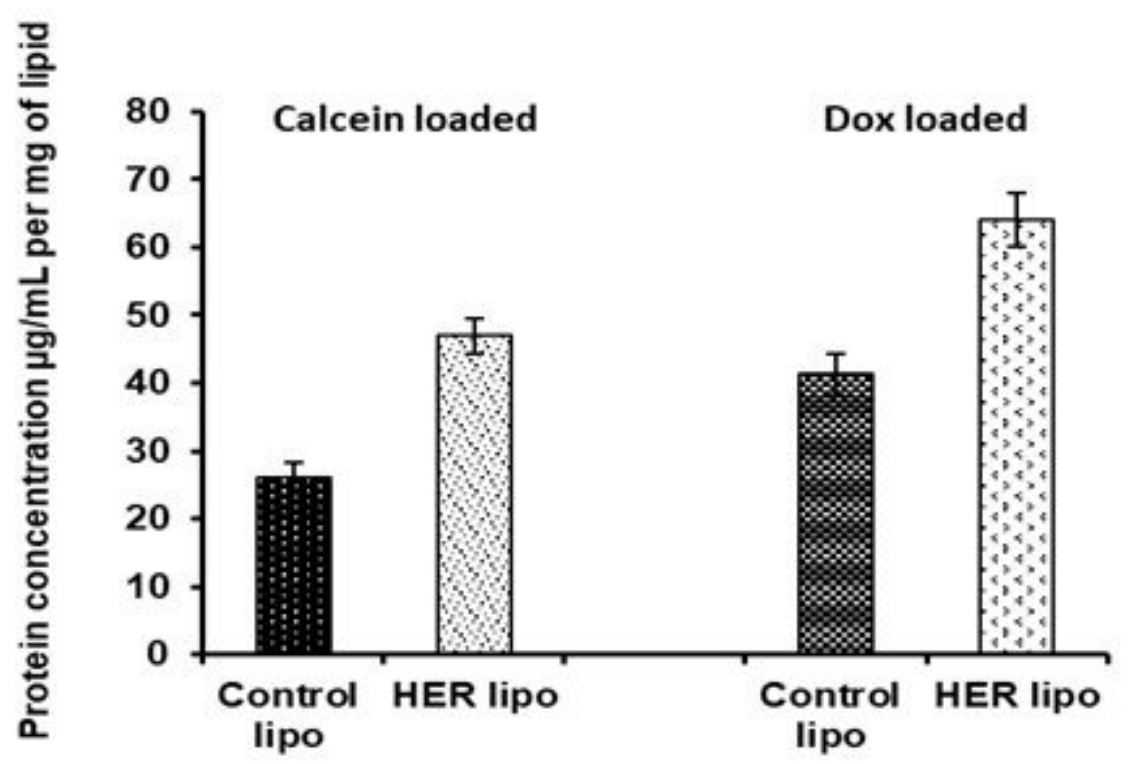

Figure 1

Protein concentrations per gm of lipids for control and immunoliposomes confirming attachment of Trastuzumab in both calcein and Dox loaded liposome.

흥

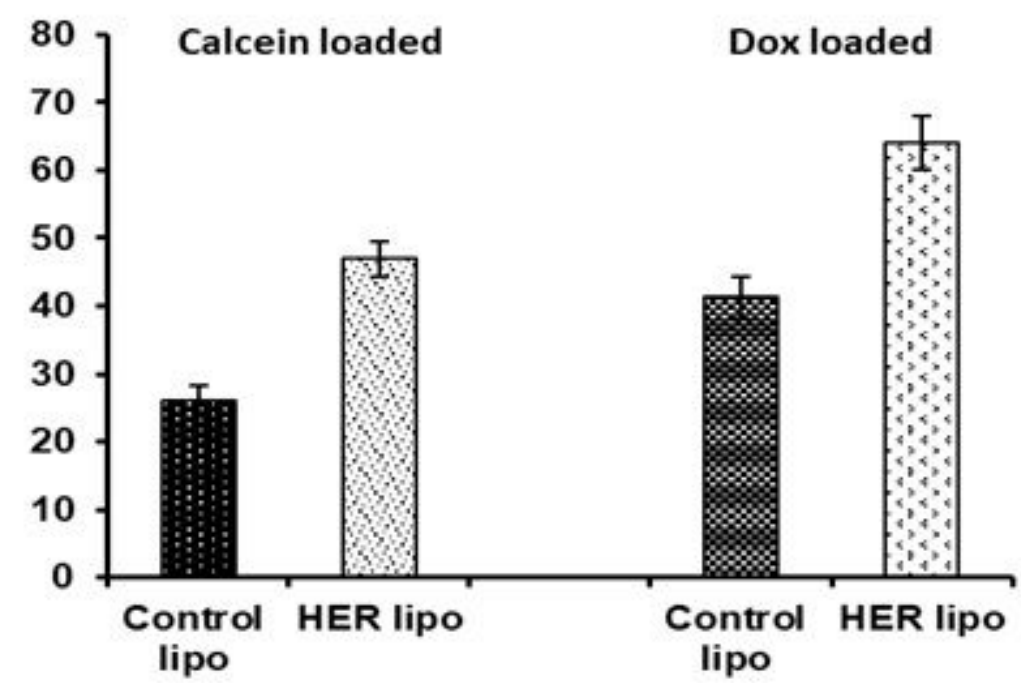

Figure 1 
Protein concentrations per gm of lipids for control and immunoliposomes confirming attachment of Trastuzumab in both calcein and Dox loaded liposome.

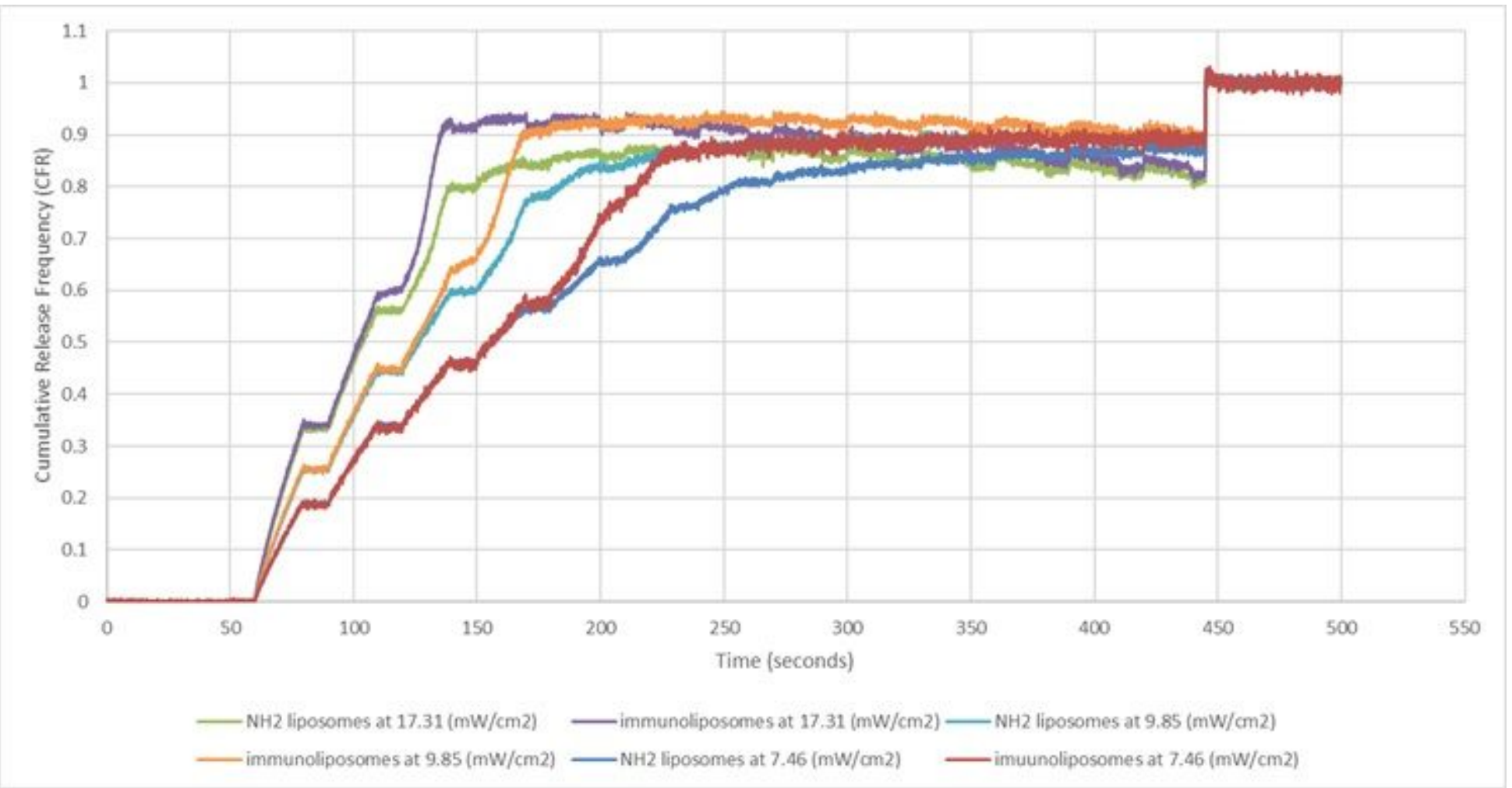

\section{Figure 2}

Normalized release profiles of 3 batches of $\mathrm{NH} 2$ and HER-liposomes loaded with calcein loaded liposomes at $7.46 \mathrm{~mW} / \mathrm{cm} 2,9.85 \mathrm{~mW} / \mathrm{cm} 2$ and $17.31 \mathrm{~mW} / \mathrm{cm} 2$.

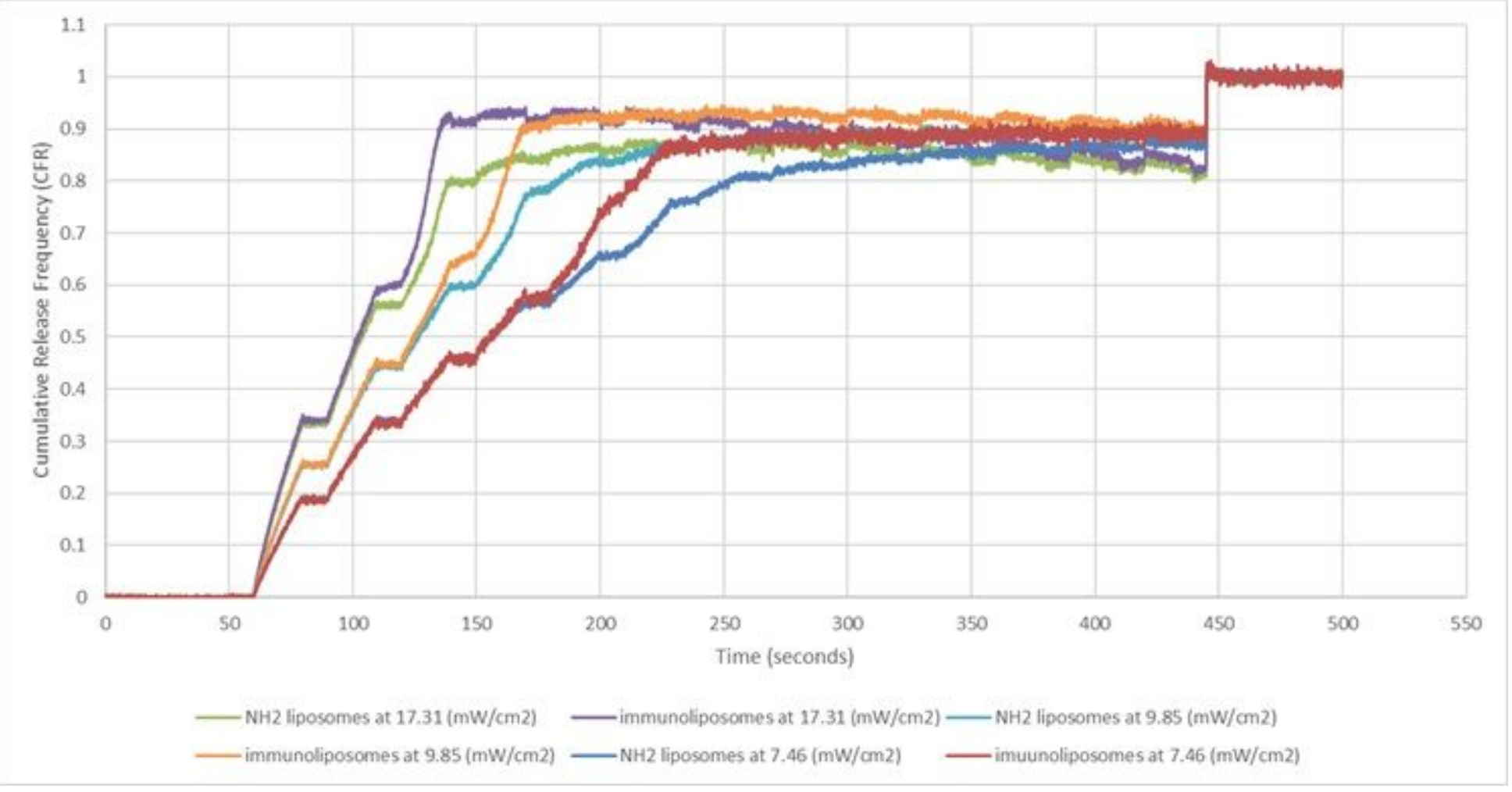


Figure 2

Normalized release profiles of 3 batches of $\mathrm{NH} 2$ and HER-liposomes loaded with calcein loaded liposomes at $7.46 \mathrm{~mW} / \mathrm{cm} 2,9.85 \mathrm{~mW} / \mathrm{cm} 2$ and $17.31 \mathrm{~mW} / \mathrm{cm} 2$.
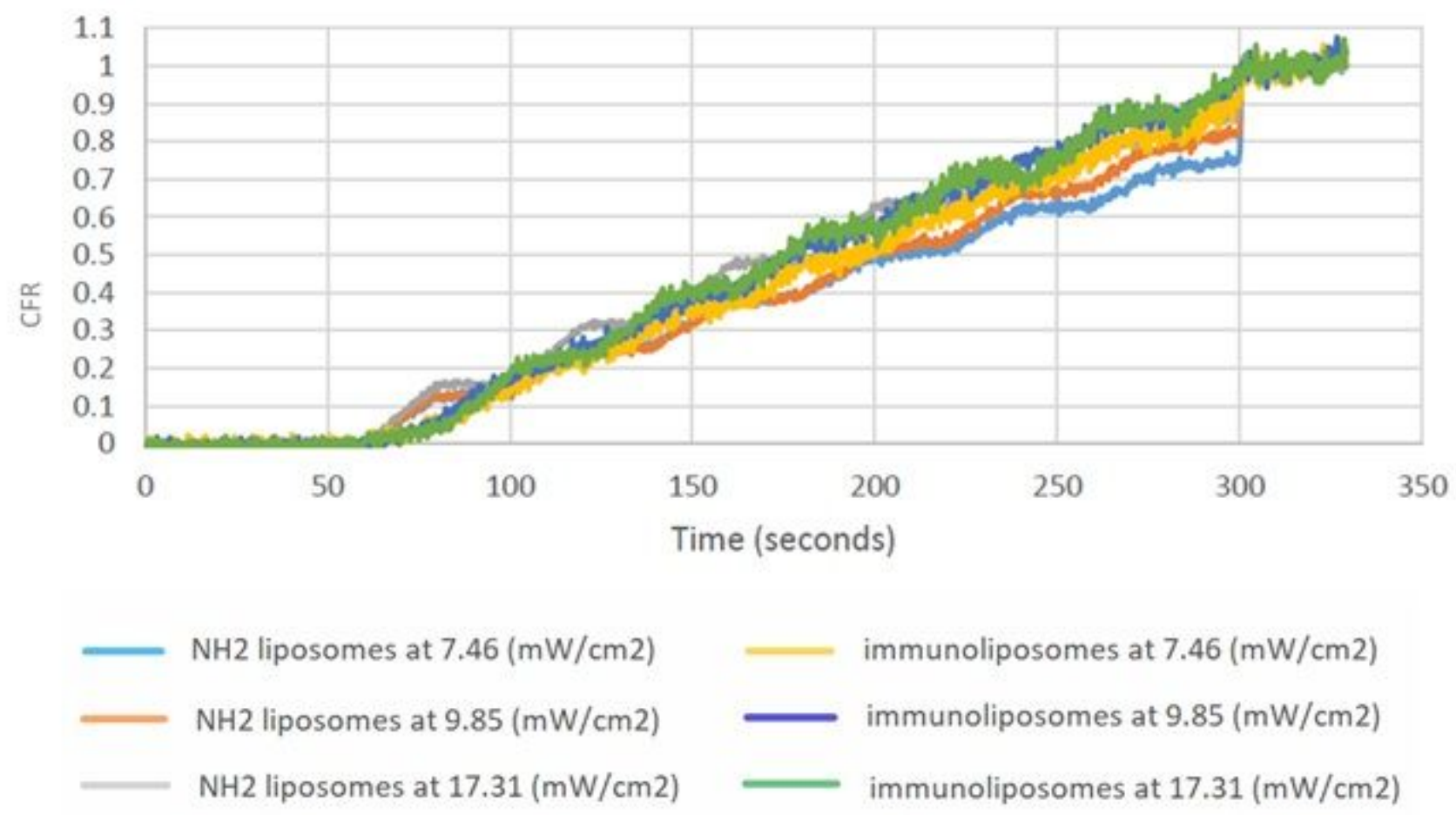

\section{Figure 3}

Normalized release profiles of 3 batches of $\mathrm{NH} 2$ and HER-liposomes loaded with doxorubicin loaded liposomes at $7.46 \mathrm{~mW} / \mathrm{cm} 2,9.85 \mathrm{~mW} / \mathrm{cm} 2$ and $17.31 \mathrm{~mW} / \mathrm{cm} 2$. 


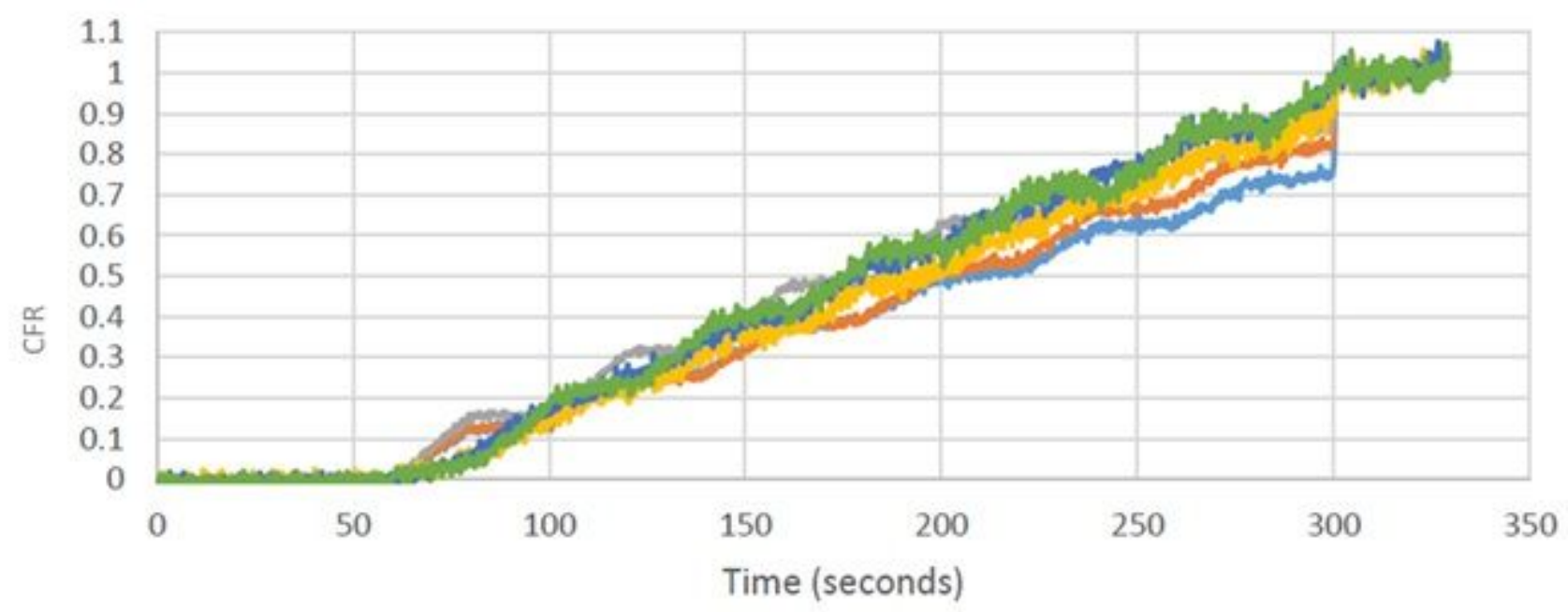

$\mathrm{NH} 2$ liposomes at $7.46(\mathrm{~mW} / \mathrm{cm} 2)$

immunoliposomes at $7.46(\mathrm{~mW} / \mathrm{cm} 2)$

$\mathrm{NH} 2$ liposomes at $9.85(\mathrm{~mW} / \mathrm{cm} 2)$

— immunoliposomes at $9.85(\mathrm{~mW} / \mathrm{cm} 2)$

$\mathrm{NH} 2$ liposomes at $17.31(\mathrm{~mW} / \mathrm{cm} 2)$

immunoliposomes at $17.31(\mathrm{~mW} / \mathrm{cm} 2)$

\section{Figure 3}

Normalized release profiles of 3 batches of $\mathrm{NH} 2$ and HER-liposomes loaded with doxorubicin loaded liposomes at $7.46 \mathrm{~mW} / \mathrm{cm} 2,9.85 \mathrm{~mW} / \mathrm{cm} 2$ and $17.31 \mathrm{~mW} / \mathrm{cm} 2$.

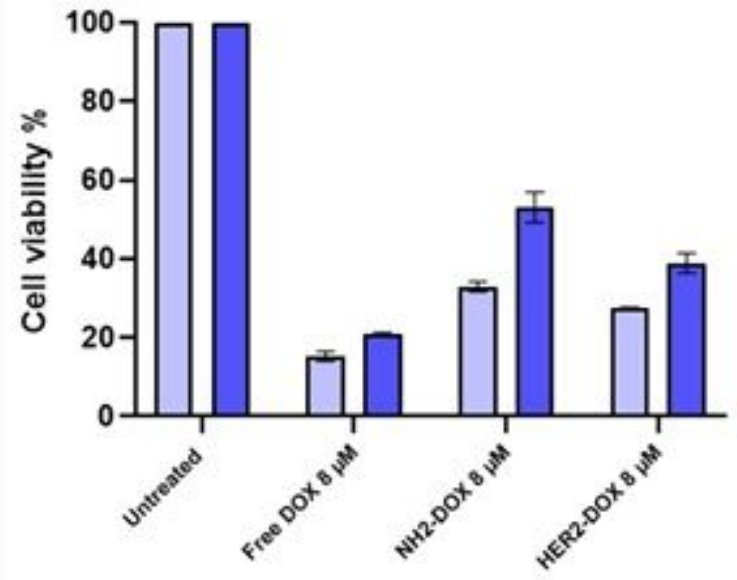

(A) SKBr-3 (HER2+ cell line)

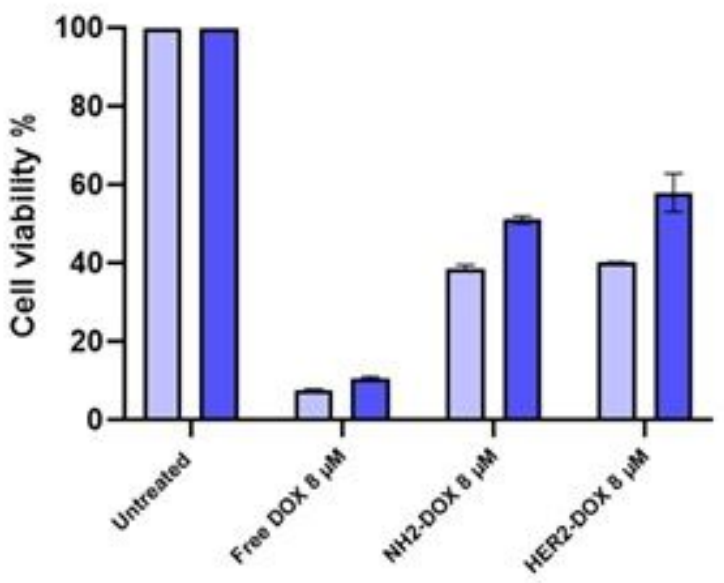

(B) MDA-MB-231 (HER2- cell line)

\section{Figure 4}

MTT results for (A) SKBr-3 cells and (B) MDA-MB-231 cells treated with free Dox, NH2-Dox liposomes and HER-DOX liposomes (DOX concentration: $8 \mu \mathrm{M}$, incubation time: $5 \mathrm{~h}$ ), in the absence and presence of US exposure. Sonicated cells were exposed to continuous LFUS in a $35-\mathrm{kHz}$ bath for $20 \mathrm{~s}$. Data are representative of three independent experiments (mean $\pm S D \%, n=3$ ). 


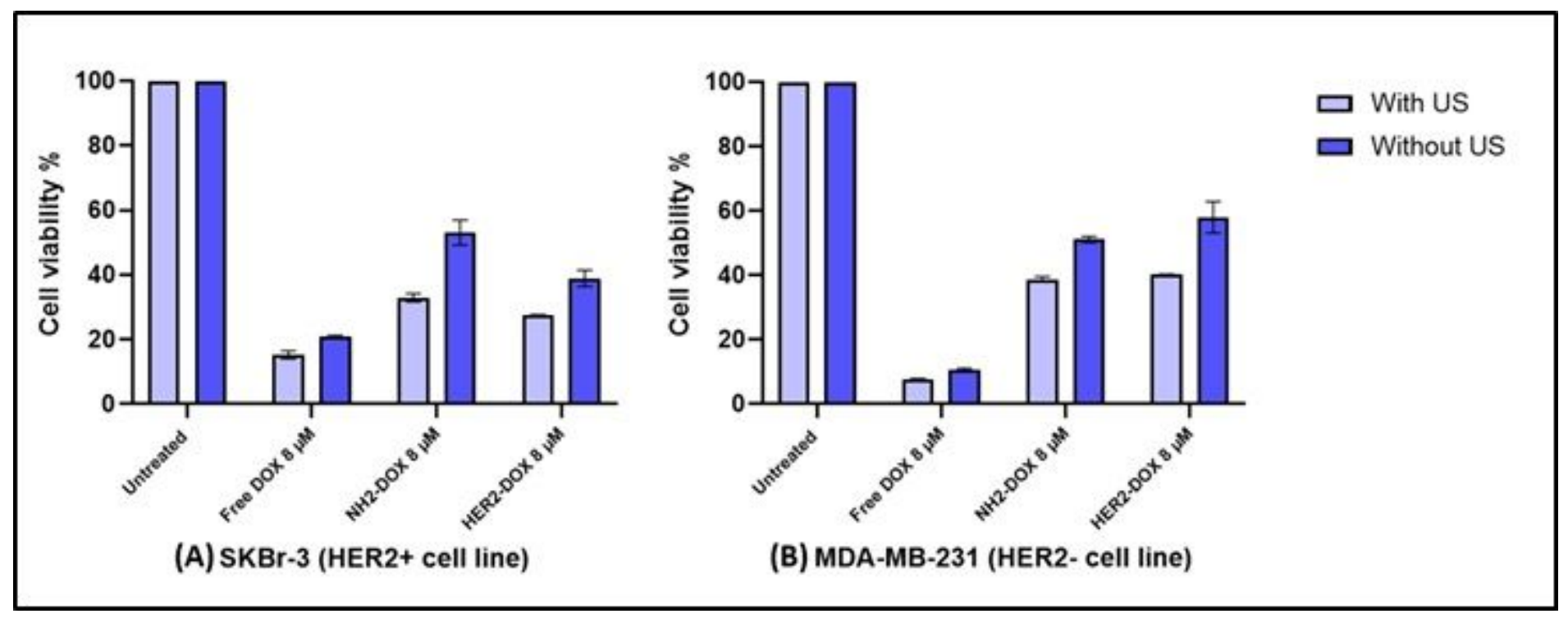

Figure 4

MTT results for (A) SKBr-3 cells and (B) MDA-MB-231 cells treated with free Dox, NH2-Dox liposomes and HER-DOX liposomes (DOX concentration: $8 \mu \mathrm{M}$, incubation time: $5 \mathrm{~h}$ ), in the absence and presence of US exposure. Sonicated cells were exposed to continuous LFUS in a $35-\mathrm{kHz}$ bath for $20 \mathrm{~s}$. Data are representative of three independent experiments (mean \pm SD \%, $n=3$ ).

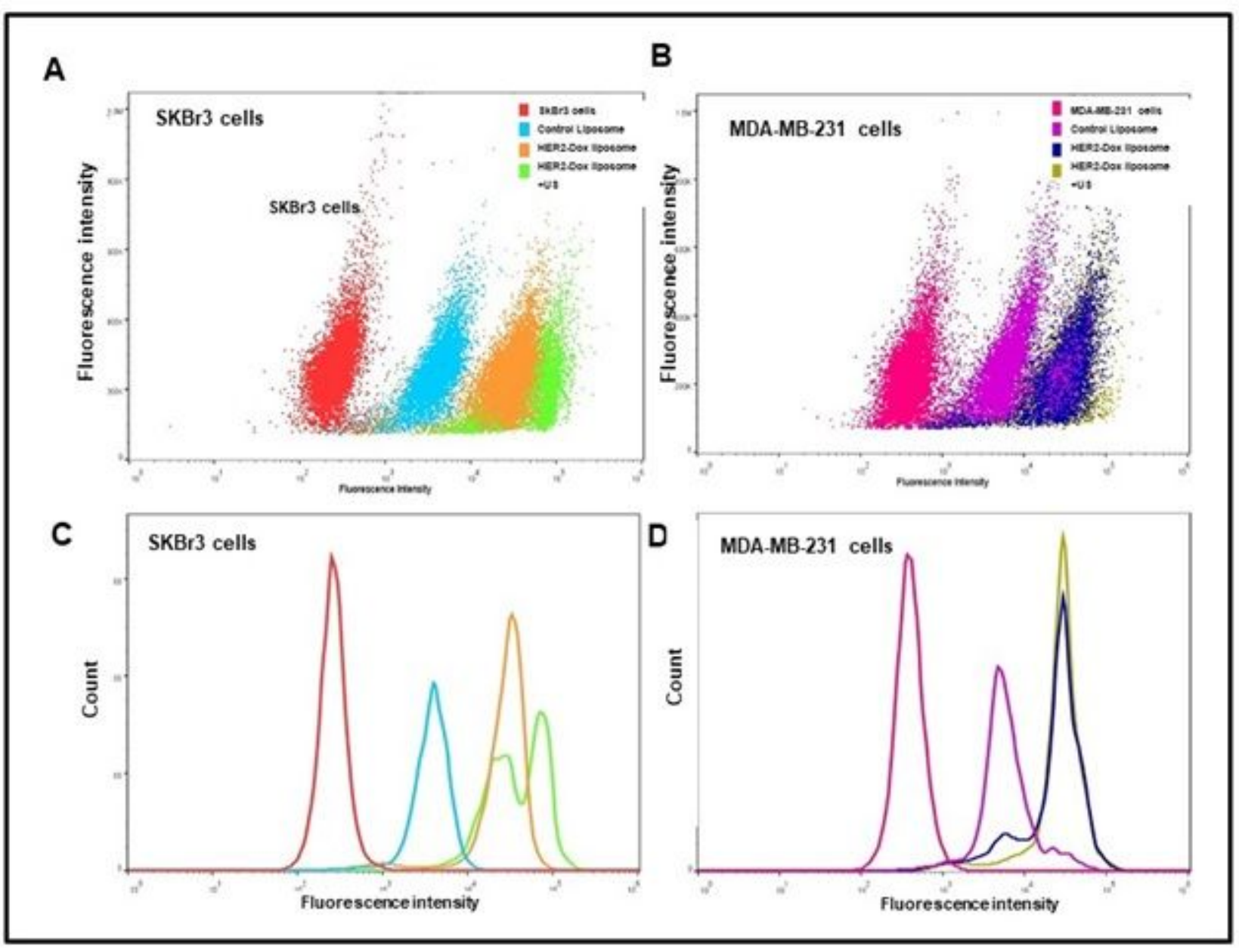

Figure 5 
Flow cytometry analysis of HER-DOX liposomes (A) SKBr3 and, (B) MDAMB-231 cells were treated with Free Dox, NH2-DOX and HER-DOX (Dox concentration: $8 \mu \mathrm{M}$ ) and incubated for 3.5h followed by LFUS at a frequency of $35 \mathrm{KHz}$ for $45 \mathrm{sec}$ at a power density of $20 \mathrm{~mW} / \mathrm{cm} 2$. Untreated cells served as a negative control for background fluorescence. An average of $1 \times 104$ cells was analyzed from each sample with the DOX fluorescence intensity showed on a four-decade log scale. Bars represent the mean fluorescence intensity $(\mathrm{MFI})$ values \pm SEM of DOX from three independent experiments between treatment groups analyzed for significance $(* p<0.05)$ using a two-tailed Student's t-test.

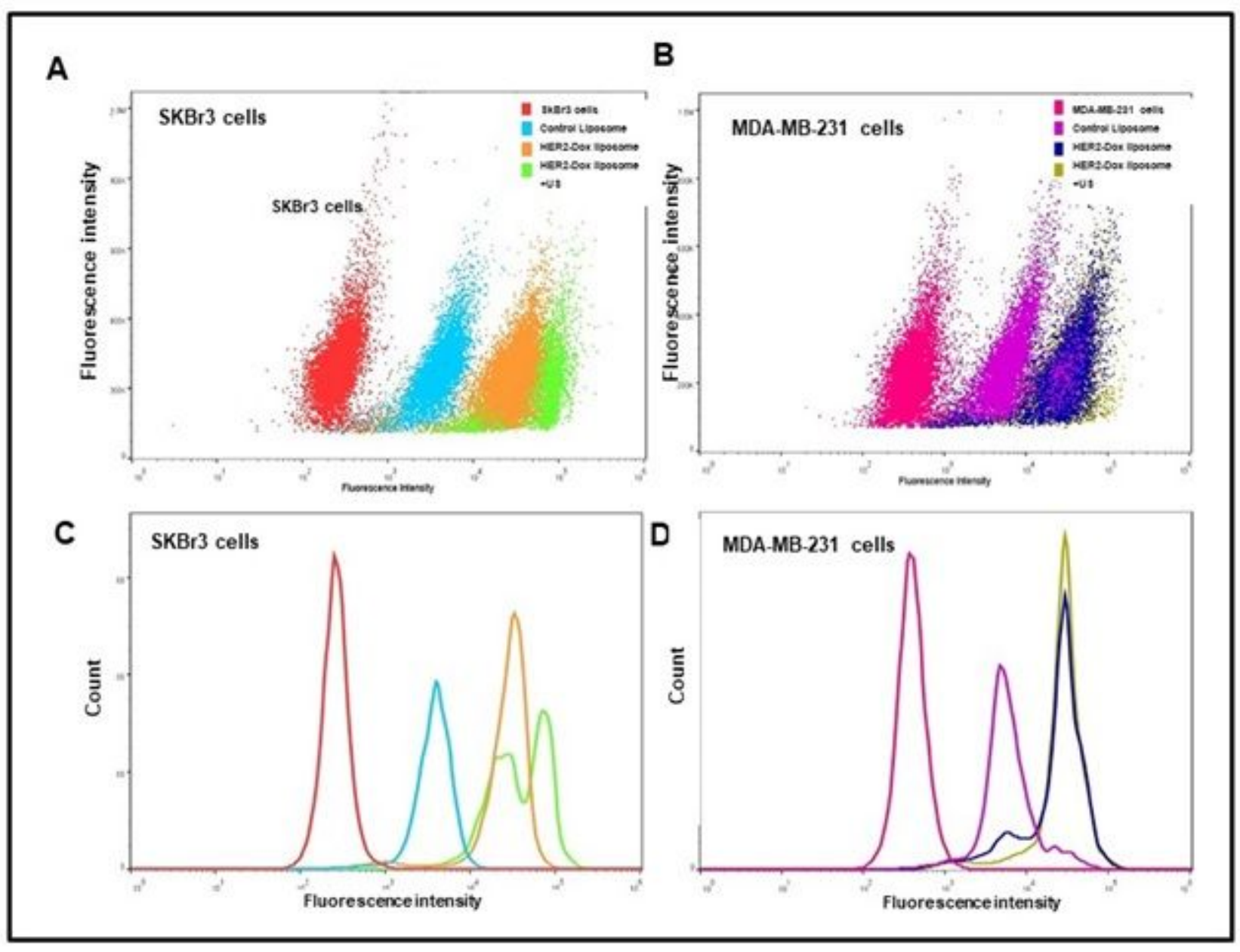

Figure 5

Flow cytometry analysis of HER-DOX liposomes (A) SKBr3 and, (B) MDAMB-231 cells were treated with Free Dox, NH2-DOX and HER-DOX (Dox concentration: $8 \mu \mathrm{M}$ ) and incubated for 3.5h followed by LFUS at a frequency of $35 \mathrm{KHz}$ for $45 \mathrm{sec}$ at a power density of $20 \mathrm{~mW} / \mathrm{cm} 2$. Untreated cells served as a negative control for background fluorescence. An average of $1 \times 104$ cells was analyzed from each sample with the DOX fluorescence intensity showed on a four-decade log scale. Bars represent the mean fluorescence intensity (MFI) values \pm SEM of DOX from three independent experiments between treatment groups analyzed for significance $(* p<0.05)$ using a two-tailed Student's t-test. 